\title{
Influence of COVID-19 disease on subarachnoid hemorrhage and intracerebral hemorrhage
}

\author{
Kafadar $\mathrm{S}^{1}$, Yucetas $\mathrm{SC}^{2}$, Gezgin $\mathrm{I}^{2}$, Kaya $\mathrm{H}^{3}$, Gulacti $\mathrm{U}^{4}$, Bilek $\mathrm{O}^{2}$
}

Adiyaman University, Department of Neurosurgery, Adiyaman, Turkey. seyhocem@hotmail.com

\begin{abstract}
This study aims to make a comparative evaluation of the change in the incidence of intracranial hemorrhage [intracerebral hemorrhage $(\mathrm{ICH})$ and subarachnoid hemorrhage $(\mathrm{SAH})$ ] cases that attended our hospital in the Covid-19 pandemic period with that of the same term one year ago. This study included 80 patients diagnosed with $\mathrm{ICH}$ and/or SAH in the period that started with the pandemic in 2020. It was determined that 51 patients had been diagnosed with $\mathrm{ICH}$ and/or SAH during the same period of 2019. A total of $131 \mathrm{ICH}$ and SAH patients (2019; $n=51,39 \%$; and $2020 ; n=80,61 \%$ ) having an average age of $64.52 \pm 7.33$ including 66 women $(50.4 \%)$ were included in the study in the nine -month follow-up periods covering the period of March-November of 2019 and 2020, respectively. It was determined that the number of patients diagnosed with $\mathrm{ICH}$ and SAH during the pandemic was higher than the number of those who attended our clinic in 2019 (80 vs 51) and that they were older (65.76 \pm 6.56 years vs $62.57 \pm 8.09$ years) $(p=0.014$ and $p=0.026$, respectively). The incidence and distribution of $\mathrm{ICH}$ and SAH among the patients were similar $(p>0.05)$. It was determined that in 1 patient, $\mathrm{ICH}$ and SAH co-existed. In the study, it was determined that among the patients treated for intracranial hemorrhage in 2020, $32.5 \%$ were diagnosed with COVID-19 as validated by positive nasopharyngeal SARS-CoV-2 PCR. The evaluation of the patients in 2020 revealed that the average age and ICH and SAH incidence in COVID-19 (+) and COVID-19 (-) patients were similar. Although increased incidence of acute intracranial hemorrhage has been observed during COVID-19 pandemic a athophysiological correlation between the two clinical presentations could not be clearly demonstrated. When rapidly progressing neurological deterioration findings are present in COVID-19 patients, existence of intracranial hemorrhage should always be considered (Tab. 2, Ref. 21). Text in PDF www.elis.sk KEY WORDS: subarachnoid hemorrhage, intracerebral hemorrhage, COVID-19.
\end{abstract}

\section{Introduction}

After the novel SARS-Cov-2 infection (COVID-19) was announced as a pandemic by the World Health Organization (WHO) (1) and Turkey reported the first official COVID-19 case around the same time (March 11, 2020), 2020 has turned into a challenging year for our country as well as the entire humanity $(2,3)$.

Intracerebral hemorrhage (ICH) and subarachnoid hemorrhage (SAH), the two major reasons for central nervous system (CNS) bleeding, are associated with certain underlying conditions such as amyloid angiopathy, hemangioma, arteriovenous malformations and coagulopathy. About 24.6 ICH and 14.9-19.6 SAH cases per 100.000 occur annually which constitute significant reasons for morbidity and mortality. Advanced age, hypertension and diabetes are correlated with negative clinic outcomes in CNS bleedings $(3,4)$.

${ }^{1}$ Department of Radiology, Adiyaman University Education and Research Hospital, Adiyaman, Turkey, ${ }^{2}$ Department of Neurosurgery, Adiyaman University Education and Research Hospital, Adiyaman, Turkey, ${ }^{3}$ Department of Cardiology, Adiyaman University Education and Research Hospital, Adiyaman, Turkey, and ${ }^{4}$ Department of Emergence Medicine, Adiyaman University Education and Research Hospital, Adiyaman, Turkey

Address for correspondence: S. Cem Yucetas, Adiyaman University, Department of Neurosurgery, Adiyaman, Turkey 416100.

Phone: +90.05066563744
The data demonstrated that human coronaviruses may be transmitted from the respiratory tract to the central nervous system by hematogenous and trans neuronal ways, causing neurological diseases and encephalitis. SARS-CoV-2 has been demonstrated in the gene sequence transferring into the cerebrospinal fluid, and the fact that it should be taken into consideration for neurological diseases has been emphasized $(5,6)$. Proof of viral infection of the endothelial cells and generalized endothelial inflammation have been reported in a number of patients diagnosed with COVID-19.

Patients experiencing intracranial hemorrhages are vulnerable against SARS-CoV-2 infection and may develop serious complications as a result of it (7). Particularly, ischemic stroke may be a symptom for COVID-19 at attendance which entails the respective risk at a rate 7.5 times higher than influenza virus infection. On the other hand, it is not clear whether SARS-CoV-2 is neurotropic or how it causes the development of neurological damage or symptoms. Similarly, it has also been reported that ICH occurs during the course of COVID-19, however significant matters such as time-wise correlation with COVID-19 infection remain unclear.

Our hospital, being a 400-bedtraining and research hospital, has a broad capacity for admitting patients diagnosed with neurosurgical pathologies, traditionally treating approximately 150 patients each year for acute and elective neurosurgical pathologies. In this study, it was aimed to compare those patients diagnosed 
with ICH and SAH who attended our clinic during a 9-month term within the COVID-19 period with those patients who had received the same diagnosis in the same period of the previous year.

\section{Materials and methods}

\section{Patients}

All patients diagnosed with SAH and ICH are admitted to the emergency department of our institution before admission to the neurosurgical intensive care unit. At this stage, all vital findings are recorded and the necessary imaging tests are conducted, which are followed by hospitalization.

\section{Data}

Demographic characteristics, medical history, clinical findings, symptoms, chest computed tomographic (CT) scan findings, laboratory findings were obtained from the electronic medical records system. The respective data was reviewed by a team of physicians specialized in their fields. Acute cerebrovascular disease covered cerebral hemorrhage and ischemic stroke diagnosed by cranial CT scan.

\section{Study method}

This study retrospectively included adult patients (age $>18$ ) diagnosed with SAH and ICH who were admitted to our institute during a 9-month period between March 1, 2020 and November 30, 2020.

The database of the hospital was used to compare our data pertaining to 2020 involving the COVID-19 pandemic period with that of the previous year for the identical time period. Patients diagnosed with SAH and ICH who attended our hospital between March 1, 2019 and November 30, 2019 (2019) were included in the study as the control group. During the aforementioned period, due to the COVID-19 pandemic, utmost care was taken about social distancing, mask use and personal isolation in Turkey.

\section{Pre-treatment planning in ICH and SAH cases}

Age, date of initial symptom, date of attendance to hospital, the World Federation of Neurosurgical Societies (WFNS) score, the Glasgow coma scale (GCS) score and existence of mydriasis were recorded for each patient at hospitalization. Evaluation of attendance with a deteriorating neurological manifestation was defined by a World Federation of Neurosurgical Societies (WFNS) score of $>3$ $(8,9)$. Information on the date of onset of COVID-19 or neurological symptoms was obtained by inquiring the patient and/or family.

\section{Pre-treatment radiological evaluation}

Existence of ICH or SAH, existence of acute hydrocephalus requiring external ventricular drainage (EVD) and the planned imaging procedures were recorded. Suitable patients developing acute hydrocephalus were given an EVD. Existence of significant levels of vasospasm in CT angiography and digital subtraction angiography (DSA) was recorded. Severe radiological manifestation was identified by the existence of ICH and/or acute hydrocephalus requiring EVD and/or existence of vasospasm during attendance $(8,9)$. All neurological symptoms were evaluated by two experienced neurosurgical specialists. The onset date of the disease was identified as the day on which the symptom was noticed. The final treatment (endovascular or microsurgical) was decided by a multidisciplinary committee made up of brain surgeons, neuroradiologists and intensive care physicians.

\section{COVID-19 diagnosis and evaluation}

All neurosurgical patients attending the emergency department of our hospital who might have been in need of surgical intervention were routinely given nasopharyngeal (NF) PCR swab testing for enabling safe perioperative evaluation. The taken swab samples were validated by real time RT-PCR test using SARS-CoV-2 nucleic acid detection kit (Device, Brand, Model, Country). The severity of COVID-19 for community-acquired pneumonia has been defined by international guidelines. Patients were treated in accordance with the instructions provided in the Updated COVID-19 Infection Treatment Guide, the Ministry of Health, and the Republic of Turkey.

\section{Ethics commitee}

The study was operated in compliance with the Declaration of Helsinki and after approved by the Local Ethics Committee of Adiyaman University (22.09.2020:2020/8-24,2020-07-21T14_12_29). The oral and written consent of all patients was obtained for the study

\section{Statistical analysis}

In the analysis of the variables, SPSS 26.0 (IBM Corporation, Armonk, New York, United States) was used. Compliance of univariate data with normal distribution was evaluated by ShapiroWilk francia test whereas homogeneity of variances was evaluated by Levene's test. In the comparison of the two independent groups in terms of quantitative data, Independent-Samples T test was used together with Bootstrapping results. For homogeneity of categorical variables, Binomial test, and in the comparison with each other, Pearson Chi-Square Test and Linear-by-Linear Association test were tested by Monte Carlo Simulation method. The quantitative variables are expressed as \pm SD (standard deviation) (Minimum/ /Maximum) in the tables whereas the categorical variables are shown as $\mathrm{n}(\%)$. The variables were examined in the confidence level of $95 \%$, and a p value lower than 0.05 was considered significant.

\section{Results}

A total of $131 \mathrm{ICH}$ and SAH patients (2019; $\mathrm{n}=51,39 \%$; and $2020 ; n=80,61 \%$ ) having an average age of $64.52 \pm 7.33$ including 66 women (50.4\%) were included in the study in the nine-month follow-up periods covering the period of March-November of 2019 and 2020, respectively. It was determined that the number of patients diagnosed with ICH and SAH during the pandemic was higher than the number of those who attended our clinic in 2019 (80 vs 51) and that they were older ( $65.76 \pm 6.56$ years vs $62.57 \pm 8.09$ years) $(p=$ 0.014 and $p=0.026$, respectively). The incidence and distribution of ICH and SAH among the patients were similar ( $p>0.05$ ). It was determined that in 1 patient, ICH and SAH co-existed (Tab. 1).

The monthly periods in which the patients attended the hospital for ICH and SAH were evaluated. It was detected that intracranial hemorrhage was most frequently observed in August $(n=18$, 
Tab. 1. Characteristics of patients diagnosed with intracranial hemorrhage in 2019 and 2020.

\begin{tabular}{|c|c|c|c|c|}
\hline & Total & 2019 & 2020 & $\mathrm{D}$ \\
\hline & $(n=131)$ & $(n=51)$ & $(n=80)$ & \\
\hline Age mean \pm SD (Min/Max) & $64.52 \pm 7.33(45 / 83)$ & $62.57 \pm 8.09(45 / 81)$ & $65.76 \pm 6.56(45 / 83)$ & $0.026^{t}$ \\
\hline & $\mathrm{n}(\%)$ & $\mathrm{n}(\%)$ & n (\%) & \\
\hline Number of cases & $131(100)$ & 51 (38.9) & $80(61.1)$ & $0.014^{b}$ \\
\hline Gender & & & & \\
\hline Female & $66(50.4)$ & $22(43.1)$ & $44(55.0)$ & $0.212^{1}$ \\
\hline Male & $65(49.6)$ & $29(56.9)$ & $36(45.0)$ & \\
\hline Subarachnoid Bleeding & & & & \\
\hline No & $71(54.2)$ & 29 (56.9) & $42(52.5)$ & $0.730^{1}$ \\
\hline Yes & $60(45.8)$ & $22(43.1)$ & $38(47.5)$ & \\
\hline Intracerebral Bleeding & & & & \\
\hline No & $59(45.0)$ & $21(41.2)$ & $38(47.5)$ & $0.589^{1}$ \\
\hline Yes & $72(55.0)$ & $30(58.8)$ & $42(52.5)$ & \\
\hline Number of cases by month & & & & \\
\hline March & $10(7.6)$ & $5(9.8)$ & $5(6.3)$ & $0.914^{1}$ \\
\hline April & $12(9.2)$ & $5(9.8)$ & $7(8.8)$ & \\
\hline May & $16(12.2)$ & $6(11.8)$ & $10(12.5)$ & \\
\hline June & 14 (10.7) & $3(5.9)$ & $11(13.8)$ & \\
\hline July & $13(9.9)$ & $6(11.8)$ & $7(8.8)$ & \\
\hline August & 18 (13.7) & $7(13.7)$ & $11(13.8)$ & \\
\hline September & $17(13.0)$ & $7(13.7)$ & $10(12.5)$ & \\
\hline October & $16(12.2)$ & $6(11.8)$ & $10(12.5)$ & \\
\hline November & 15 (11.5) & $6(11.8)$ & 9 (11.3) & \\
\hline
\end{tabular}

'Independent Samples t-test (Bootstrap), ${ }^{\mathrm{b}}$ One Sample Binomial test(Monte Carlo), ${ }^{1}$ Linear-by-Linear Association Test(Monte Carlo, Exact), SS.: Standard deviation, Min.: Minimum, Max.: Maximum

Tab. 2. Evaluation of patients diagnosed with intracranial hemorrhage in terms of COVID-19.

\begin{tabular}{|c|c|c|c|}
\hline & \multicolumn{3}{|c|}{ COVID-19 (2020 data) } \\
\hline & No $(n=54,67.5 \%)$ & Yes $(n=26,32.5 \%)$ & $\mathrm{p}$ \\
\hline \multirow[t]{2}{*}{ Age mean \pm SD (Min/Max) } & $66.69 \pm 6.35(45 / 83)$ & $63.85 \pm 6.68(48 / 75)$ & $0.572^{\mathrm{t}}$ \\
\hline & $\mathrm{n}(\%)$ & $\mathrm{n}(\%)$ & \\
\hline \multicolumn{4}{|l|}{ Gender } \\
\hline Female & $29(53.7)$ & $15(57.7)$ & $0.512^{\mathrm{p}}$ \\
\hline Male & $25(46.3)$ & $11(42.3)$ & \\
\hline \multicolumn{4}{|l|}{ Subarachnoid hemorrhage } \\
\hline No & $29(53.7)$ & $13(50.0)$ & $0.665^{p}$ \\
\hline Yes & $25(46.3)$ & $13(50.0)$ & \\
\hline \multicolumn{4}{|l|}{ Intracerebral bleeding } \\
\hline No & $25(46.3)$ & $13(50.0)$ & $0.661^{p}$ \\
\hline Yes & $29(53.7)$ & $13(50.0)$ & \\
\hline \multicolumn{4}{|l|}{ Month } \\
\hline March & $4(7.4)$ & $1(3.8)$ & $0.952^{\mathrm{ff}}$ \\
\hline April & $5(9.3)$ & $2(7.7)$ & \\
\hline May & $7(13.0)$ & $3(11.5)$ & \\
\hline June & $7(13.0)$ & $4(15.4)$ & \\
\hline July & $5(9.3)$ & $2(7.7)$ & \\
\hline August & $7(13.0)$ & $4(15.4)$ & \\
\hline September & $8(14.8)$ & $2(7.7)$ & \\
\hline October & $6(11.1)$ & $4(15.4)$ & \\
\hline November & $5(9.3)$ & $4(15.4)$ & \\
\hline
\end{tabular}

t Independent Samples t test (Bootstrap), b One Sample Binomial test (Monte Carlo), l Linear-by-Linear Association Test( Monte Carlo, Exact), SD.: Standard Deviation

$13.7 \%$ ) and September ( $\mathrm{n}=17,13 \%)$. As for 2020 which included the COVID-19 pandemic process, it was detected that the most frequent attendances to hospital have been in June and September $(n=11,13.8)$. On the other hand, no significant difference was found in terms of monthly number of patient attendance (Tab. 1).

In the study, it was determined that among the patients treated for intracranial hemorrhage in 2020, $22.5 \%$ were diagnosed with
COVID-19 as validated by positive nasopharyngeal SARS-CoV-2 PCR. The evaluation of the patients in 2020 revealed that the average age and ICH and SAH incidence of COVID-19 (+) and COVID-19 (-) patients were similar. No differences were detected in the monthly distribution of the patients during the COVID-19 pandemic in terms of PCR $(+)$ rates $(\mathrm{p}>0.05)$ (Tab. 2).

\section{Discussion}

Due to the global spread of the novel coronavirus strain (SARS-CoV-2) which causes the respiratory disease called COVID-19, there has been a growing interest in the medical world in its common effects. Specifically, it has been getting clearer that in many patients, neurological symptoms constitute the initial symptoms of COVID-19. Although clinical data is not sufficient, there has nevertheless been heavy concern about the fact that COVID-19 may increase or trigger hemorrhagic stroke risk, especially in elderly patients $(10,11)$. It is probable that subarachnoid hemorrhage developing in a patient may be due to the increase in the intraluminal pressure of the radiculo-medullary veins that pass into the subarachnoid space. Latest studies have shown that due to severe coughing, these veins may be subject to spontaneous pressure increase and rupture which in turn may lead to spontaneous subarachnoid hemorrhage. The results of our study have shown a significant increase in the number of patients diagnosed with SAH and ICH who attended our clinic in 2020.

Although susceptibility to thrombosis is well known in COVID-19, there are a considerable number of case reports connected to hemorrhage affecting multiple organ systems including the CNS. The causal pathophysiological process has not yet been fully understood, and hemorrhage secondary to microthrombosis, cytokine storm (12), endotheliosis (13), disseminated intravascular coagulation with underlying excessive vascular permeability and vasculitis (14), have been held responsible. In a recently published neuroimaging study by Mahammedi et al (15), it has been shown that in COVID-19 patients, imaging is rich in acute ischemic infarct and intracranial hemorrhage. Following several proofs in the current literature about the correlation between COVID-19 and cerebrovascular disease, there is a compulsory need for defining such correlations and outcomes. Striking proofs have shown that in adults, COVID-19 
causes multisystem organ involvement including cerebrovascular occurrences such as the central nervous system (CNS) and intracranial hemorrhage (16-18).

Sharifi-Razavi et al (19) reported an intracranial hemorrhage case developing in a 79-year-old, COVID-19-positive male who had no previous hypertension history or anticoagulant use. In a case series consisting of five patients, Benger et al. contributed to the discussions on the COVID-19 and intracerebral hemorrhage association (20). In this series, particularly the clinical challenges occurring in the management of the thrombotic complications of COVID-19 have been emphasized. In three out of the five patients in the series, anticoagulation was used in the pre-ICH treatment dosage whereas the remaining two patients took a prophylactic LMWH, which has been considered as a significant confounding factor in interpreting the correlation of the clinical results with COVID-19. In our study, the increased hemorrhage incidence during the pandemic in patients of advanced age having negative outcome risk correlated with COVID-19 and intracranial hemorrhage suggests that elderly patients go through a quite fragile process.

Due to its retrospective nature, this study entails certain limitations in evaluating the COVID-19-related clinical findings of the cases with intracerebral hemorrhage, especially in the pandemic period, comprising detailed evaluation of the COVID-19-related clinical outcomes and intracranial hemorrhage etiologies of the patients, and difficulty in accessing detailed data about postoperative follow-up. The detection of simultaneous COVID-19 in 32.5\% of the patients treated in our clinic for intracranial hemorrhage in 2020 has been determined to be quite high in proportion to the COVID-19 incidence reported in the literature. This may have been a result of the inclusion of asymptomatic patients as the patients were routinely given preoperative SARS-CoV-2 PCR validation, and the patients getting infected during follow-up.

In the light of the present lack of evidence, it may remain merely theoretical whether this situation exists in certain rapidly developing pulmonary deficiency in COVID-19 patients [21]. We consider that in order to shed light on the real effects, mechanisms and systemic results of CNS involvement in COVID-19 patients, other pathological studies such as necropsy, or more suitably in view of the current health emergency, even minimally invasive brain biopsies may be requested.

\section{Conclusions}

As a result, although increased incidence of acute intracranial hemorrhage has been observed during COVID-19 pandemic a pathophysiological correlation between the two clinical presentations could not be clearly demonstrated. When rapidly progressing neurological deterioration findings are present in COVID-19 patients, existence of ICH should always be considered.

\section{References}

1. World Health Organization (WHO). Coronavirus disease 2019 Situation Report 51 11th March 2020. World Heal Organ 2020; 2019 (March): 2633.

2. Pope JE. What Does the COVID-19 Pandemic Mean for Rheumatology Patients? Curr Treat Options Rheumatol 2020; 6 (2): 71-74.
3. Ma L, Zhang S, Li Z, Wu C-X, Wang Z, Zhan L et al. Morbidity After Symptomatic Hemorrhage of Cerebral Cavernous Malformation: A Nomogram Approach to Risk Assessment. Stroke 2020; 51 (10): 2997-3006.

4. Hughes JD, Bond KM, Mekary RA, Dewan MC, Rattani A, Baticulon R et al. Estimating the Global Incidence of Aneurysmal Subarachnoid Hemorrhage: A Systematic Review for Central Nervous System Vascular Lesions and Meta-Analysis of Ruptured Aneurysms. World Neurosurg 2018; 115: 430-447.

5. GuptaA, Madhavan MV, Sehgal K, Nair N, Mahajan S, Sehrawat TS et al. Extrapulmonary manifestations of COVID-19. Nat Med 2020; 26(7): 1017-1032.

6. Al Saiegh F, Ghosh R, Leibold A, Avery MB, Schmidt RF, Theofanis T et al. Status of SARS-CoV-2 in cerebrospinal fluid of patients with COVID-19 and stroke. J Neurol Neurosurg Psychiatry 2020; 91 (8): 846-848.

7. Melmed KR, Cao M, Dogra S, Zhang R, Yaghi S, Lewis A et al. Risk factors for intracerebral hemorrhage in patients with COVID-19. J Thromb Thrombolysis 2020; (0123456789).

8. Hemphill JC 3rd, Greenberg SM, Anderson CS, Becker K, Bendok BR, Cushman M et al. Guidelines for the Management of Spontaneous Intracerebral Hemorrhage: A Guideline for Healthcare Professionals From the American Heart Association/American Stroke Association. Stroke 2015; 46 (7): 2032-2060.

9. Hijdra A, Brouwers P, Vermeulen M, Gijn J Van. Grading the amount of blood on computed tomograms after subarachnoid hemorrhage. Stroke 1990; 21 (8): 1156-1161.

10. Wang H, Tang X, Fan H, Luo Y, Song Y, Xu Y et al. Potential mechanisms of hemorrhagic stroke in elderly COVID-19 patients. Aging (Albany NY) 2020; 12 (11): 10022-10034.

11. Hanafi R, Roger PA, Perin B, Kuchcinski G, Deleval N, Dallery F et al. COVID-19 Neurologic Complication with CNS Vasculitis-Like Pattern. Am J Neuroradiol 2020; 41 (8): 1384-1387.

12. Vinayagam S, Sattu K. SARS-CoV-2 and coagulation disorders in different organs. Life Sci 2020; 260 (8): 118431.

13. Varga Z, Flammer AJ, Steiger $\mathbf{P}$, Haberecker $M$, Andermatt R, Zinkernagel AS et al. Endothelial cell infection and endotheliitis in COVID-19. Lancet 2020; 395 (10234): 1417-1418.

14. Vacchi C, Meschiari M, Milic J, Marietta M, Tonelli R, Alfano G et al. COVID-19-associated vasculitis and thrombotic complications: from pathological findings to multidisciplinary discussion. Rheumatology (Oxford) 2020; 59 (12): e147-150.

15. Mahammedi A, Saba L, Vagal A, Leali M, Rossi A, Gaskill M et al. Imaging of Neurologic Disease in Hospitalized Patients with COVID-19: An Italian Multicenter Retrospective Observational Study. Radiology 2020; 297 (2): E270-273.

16. Reddy ST, Garg T, Shah C, Nascimento FA, Imran R, Kan P et al. Cerebrovascular Disease in Patients with COVID-19: A Review of the Literature and Case Series. Case Rep Neurol 2020; 12 (2): 199-209.

17. Mao L, Wang M, Chen S, He Q, Chang J, Hong CD et al. Neurological manifestations of hospitalized patients with COVID-19 in Wuhan, China: A retrospective case series study. medRxiv 2020.

18. Fotuhi M, Mian A, Meysami S, Raji CA. Neurobiology of COVID-19. J Alzheimer's Dis 2020; 76 (1): 3-19.

19. Sharifi-Razavi A, Karimi N, Rouhani N. COVID-19 and intracerebral haemorrhage: causative or coincidental? New Microbes New Infect 2020; 35: 0-1.

20. Benger M, Williams O, Siddiqui J, Sztriha L. Intracerebral haemorrhage and COVID-19: Clinical characteristics from a case series. Brain Behav Immun 2020; 88: 940-944.

21. Altschul DJ, Unda SR, de La Garza Ramos R, Zampolin R, Benton J, Holland R et al. Hemorrhagic presentations of COVID-19: Risk factors for mortality. Clin Neurol Neurosurg 2020; 198: 106112.

Received September 3, 2021. Accepted September 21, 2021. 